\title{
STUMBLING UPON PALATOGINGIVAL GROOVE: A CASE REPORT
}

\author{
Sujan Thapa ${ }^{1}$, Vanita Gautam ${ }^{1}$, Snigdha Shubham ${ }^{1}$, Manisha Nepal ${ }^{1}$, Kriti Shrestha ${ }^{1}$
}

\begin{abstract}
Palatogingival grooves (PGG) are developmental malformations infamous for precipitating endodontic-periodontal lesions. Pertaining to their discreet occurrence, variation in groove depth and funnel like shape, adherence of plaque and calculus is apparent thereby jeopardizing the periodontium and pulp. A case of palatogingival groove in maxillary left lateral incisor was diagnosed and managed with endodontic treatment followed by open flap debridement to seal the groove with biodentine and fill the defect with bone graft. On follow up, the periapical lesion and the periodontal pocket were successfully resolved.
\end{abstract}

\section{KEYWORDS}

Biodentine, Endodontic-periodontal lesion, Incisor, Palatogingival Groove

1. Department of Conservative Dentistry and Endodontics, UCMS, Bhairahawa, Nepal

DOI: https://doi.org/10.3126/jucms.v9i02.42023

\author{
For Correspondence \\ Dr. Sujan Thapa \\ Junior Resident \\ Department of Conservative Dentistry and Endodontics \\ Bhairahawa, Nepal \\ Email: thesujan24@gmail.com
}




\section{INTRODUCTION}

A palatal groove is a developmental groove in a root that, when present, is usually found on the lingual aspect of maxillary teeth. ${ }^{1}$ First described by Black in 1908 as a radicular groove ${ }^{2}$, the term Palatogingival groove (PGG) was proposed by Lee et al in $1968 .{ }^{3}$ It is also called Vertical Developmental Radicular Groove, Syndesmocoronoradicular Tooth and Interruption groove. ${ }^{4}$ Its prevalence is rare ranging from $2.8 \%-8.5 \%{ }^{5}$ Its etiopathogenesis is controversial, considered to arise due to genetic alteration or as an infolding of enamel epithelium and Hertwigs Epithelial Root Sheath during odontogenesis. ${ }^{6}$ Clinically, PGG presents as V shaped indentation starting from the cingulum extending apically to varying distances along the root surface. It is mostly associated with deep periodontal pockets, alveolar bone loss and pulp necrosis, hence resulting combined endo-perio lesions.

\section{CASE REPORT}

A 28 year-old female patient reported with the chief complain of pain in the left upper front tooth for 3 days. The pain had sudden onset, rapid progression, throbbing nature and was relieved on medication. There was no apparent history of trauma on the offending tooth. On examination, the overall oral hygiene was good. The tooth was tender on percussion and the mobility was Grade II. It showed no response on Electric pulp Test (EPT) and Cold Test with Endo Ice. Radiograph revealed widened Periodontal Ligament (PDL) space along the entire root length and a round diffuse radiolucency surrounding the apex of the root. A thin radiolucent line adjacent to the root canal was evident which created a diagnostic dilemma. On clinical reassessment, a groove was noticed on the palatal aspect starting at the cingulum and extending to the root which on corelating with the radiograph was the radiolucent parapulpal line, indeed a PGG. A periodontal pocket of $10 \mathrm{~mm}$ was recorded in midpalatal aspect of the root. A final diagnosis of Pulp Necrosis; Acute Periapical Abscess wrt 22 was made. Root Canal Therapy (RCT) followed by open flap debridement coupled with simple closure of the palatogingival groove with Biodentine and filling the bone defect with bone graft was planned.

An endodontic access was opened in the palatal aspect of 22 using round carbide bur and endo Z bur (Dentsply) which revealed a single canal orifice. After establishing the working length, chemomechanical preparation was done with conventional hand filing. Aqueous $\mathrm{Ca}(\mathrm{OH})_{2}$ (Maarc) intracanal medicament was placed and the patient was kept on follow-up. On the third visit, she was asymptomatic and the mobility had significantly reduced to normal limits but there was persistence of the pocket. Obturation was done with GP cones and ZOE sealer followed by Glass Ionomer Cement. After presurgical rinse with $0.12 \%$ CHX mouthwash, $2 \%$ Lignocaine with 1:200000 dilution was administered. On the palatal aspect, a full thickness mucoperiosteal flap was raised to expose the bony defect. The PGG was isolated and found to extend beyond $2 / 3^{\text {rd }}$ of the root. A three-wall bony defect at the root apex was encountered. The diseased granulation tissue was removed to make the soft tissues more conducive to regeneration. Biodentine (Septodont) was used to seal the groove. A bone graft (Sybograf*Plus) was used to fill the bone defect. Finally, the flaps were approximated with simple interrupted sutures using mersilk 4-0. At 1 year follow up, the lesion was healed.
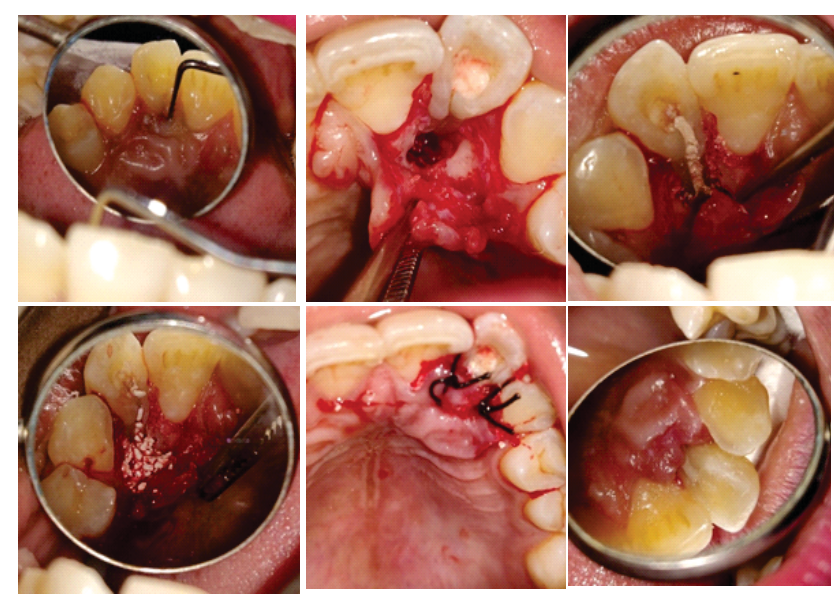

Fig 1. A) The palatal view of left maxillary incisor showing a groove arising from cingulum and reaching the root associated with a $10 \mathrm{~mm}$ pocket $B$ ) The reflection of the flap allowing a direct visualization of the palatoradicular groove and the intrabony defect $C$ ) Sealing the palato gingival groove with Biodentine $D$ ) Filling the bone defect with a bone graft E) Flaps approximated with 3-0 mersilk suture F) Intraoral examination at 10 day follow up

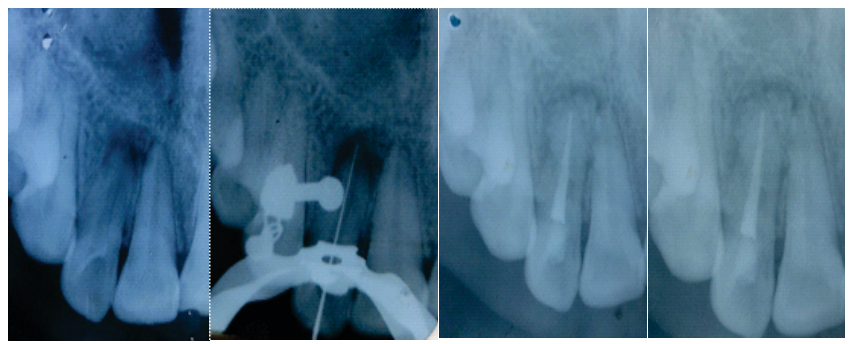

Fig 2. A) IOPAR wrt 22 revealing a periapical lesion and a radiolucent parapulpal line B) Working Length Determination wrt 22 C) Radiograph immediately after flaps were approximated D) Radiograph at 1 year follow up 


\section{DISCUSSION}

PGG is a rare morphological aberrancy that usually begins at or near the tooth cingulum region extending apically from the cementoenamel junction (CEJ) along the root surface of maxillary anterior teeth. Though parapulpal radiolucent lines might assist in diagnosing, the key to final diagnosis is to detect a notch at or near the cingulum of the crown. ${ }^{7}$ PGG predisposes to endodontic or periodontal disease or combined endodontic-periodontal disease, presenting asymptomatically or with diverse symptoms. Prognosis is dependent on the groove location, type and accessibility of the defect. ${ }^{8}$ There are various treatment modalities available for these conditions, ranging from nonsurgical to surgical procedures such as RCT, crown restoration for groove elimination, odontoplasty (saucerization), intentional replantation, Open Flap Debridement (OFD) and Guided Tissue Regeneration (GTR). ${ }^{9}$ When the groove is entirely located in the crown of the tooth, terminating at the CEJ and when it is shallow, the prognosis can be estimated as good since there will be neither a deep bony defect nor pulpal damage. Mild PGG can be treated with Saucerization that involves grinding the groove to the level of the crestal bone with a rotary cutting and polishing instrument. With deeper grooves extended onto the root surface and substantial periodontal destruction, a flap procedure, including curettage of granulation tissue and root planing needs to be undertaken. The grooves can be sealed with dental materials like GIC, composite, mineral trioxide aggregate and biodentine. ${ }^{10}$ For the periodontal defect, GTR can be performed to fill the osseous defect with bone graft material and the barrier membrane to provide epithelial exclusion, thus permitting regeneration of the cementum, PDL and alveolar bone. An alternative option is intentional replantation wherein the tooth is extracted as atraumatically as possible, endodontically treated and groove sealed outside the oral cavity then reimplanted.

\section{CONCLUSION}

A diagnostic dilemma always exists due to varied clinical presentation of PGG often leading to delayed treatment and undesired extraction of the tooth. Yet with recent treatment modalities, complete resolution of the lesion and survival of a tooth can be ensured.

\section{REFERENCES}

1. Eleazer P, Glickman G, McClanahan S, Webb T, Jusrman B. Glossary of endodontic terms. Editorial AAE: Chicago. 2012.

2. Black GV. Pathology of the hard tissues of the teeth, oral diagnosis. Medico Dental Publishing Company; 1936.
3. Lee KW, Lee EC, Poon KY. Palato-gingival grooves in maxillary incisors. A possible predisposing factor to localised periodontal disease. British dental journal. 1968 Jan 2;124(1):14-8.

4. Kim HJ, Choi Y, Yu MK, Lee KW, Min KS. Recognition and management of palatogingival groove for tooth survival: a literature review. Restorative dentistry \& endodontics. 2017 May;42(2):77.

5. Bharti R, Chandra A, Tikku AP, Arya D. Palatogingival groove: A cause for periapical infection. International Journal of Prosthodontics and Restorative Dentistry. 2012 Apr 4;2(2):615.

6. Cho YD, Lee JE, Chung Y, Lee WC, Seol YJ, Lee YM, Rhyu IC, $\mathrm{Ku}$ Y. Collaborative management of combined periodontalendodontic lesions with a palatogingival groove: a case series. Journal of endodontics. 2017 Feb 1;43(2):332-7.

7. Devi BP, Das A, Kundu R, Bagchi S, Pal AK. The silent killer caught white handed: An interdisciplinary approach to manage a rare case of PGG associated Perio-endo lesion.

8. Gu YC. A micro-computed tomographic analysis of maxillary lateral incisors with radicular grooves. Journal of Endodontics. 2011 Jun 1;37(6):789-92.

9. Robison SF, Cooley RL. Palatogingival groove lesions: recognition and treatment. General dentistry. $1988 \mathrm{Jul}$ 1;36(4):340-2.

10. Sharma S, Deepak P, Vivek S, Dutta SR. Palatogingival groove: recognizing and managing the hidden tract in a maxillary incisor: a case report. Journal of international oral health: JIOH. 2015 Jun; 7(6):110. 